\title{
A new genome-scale metabolic model of Corynebacterium glutamicum and its application
}

Yu Zhang ${ }^{1,2}$, Jingyi Cai ${ }^{3}$, Xiuling Shang ${ }^{1}$, Bo Wang ${ }^{1,2}$, Shuwen Liu' ${ }^{1}$, Xin Chai ${ }^{1,2}$, Tianwei Tan ${ }^{3}$, Yun Zhang ${ }^{\text {* }}$ and Tingyi Wen ${ }^{1,4^{*}}$

\begin{abstract}
Background: Corynebacterium glutamicum is an important platform organism for industrial biotechnology to produce amino acids, organic acids, bioplastic monomers, and biofuels. The metabolic flexibility, broad substrate spectrum, and fermentative robustness of C. glutamicum make this organism an ideal cell factory to manufacture desired products. With increases in gene function, transport system, and metabolic profile information under certain conditions, developing a comprehensive genome-scale metabolic model (GEM) of C. glutamicum ATCC13032 is desired to improve prediction accuracy, elucidate cellular metabolism, and guide metabolic engineering.
\end{abstract}

Results: Here, we constructed a new GEM for ATCC13032, iCW773, consisting of 773 genes, 950 metabolites, and 1207 reactions. Compared to the previous model, iCW773 supplemented 496 gene-protein-reaction associations, refined five lumped reactions, balanced the mass and charge, and constrained the directionality of reactions. The simulated growth rates of $C$. glutamicum cultivated on seven different carbon sources using iCW773 were consistent with experimental values. Pearson's correlation coefficient between the iCW773-simulated and experimental fluxes was 0.99 , suggesting that iCW773 provided an accurate intracellular flux distribution of the wild-type strain growing on glucose. Furthermore, genetic interventions for overproducing L-lysine, 1,2-propanediol and isobutanol simulated

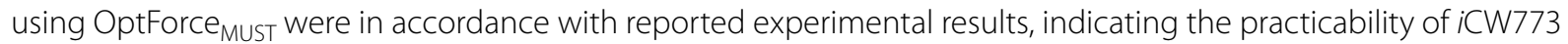
for the design of metabolic networks to overproduce desired products. In vivo genetic modifications of iCW773predicted targets resulted in the de novo generation of an L-proline-overproducing strain. In fed-batch culture, the engineered C. glutamicum strain produced $66.43 \mathrm{~g} / \mathrm{L}$ L-proline in $60 \mathrm{~h}$ with a yield of $0.26 \mathrm{~g} / \mathrm{g}$ (L-proline/glucose) and a productivity of $1.11 \mathrm{~g} / \mathrm{L} / \mathrm{h}$. To our knowledge, this is the highest titer and productivity reported for L-proline production using glucose as the carbon resource in a minimal medium.

Conclusions: Our developed iCW773 serves as a high-quality platform for model-guided strain design to produce industrial bioproducts of interest. This new GEM will be a successful multidisciplinary tool and will make valuable contributions to metabolic engineering in academia and industry.

Keywords: Corynebacterium glutamicum, Genome-scale metabolic model, L-proline production, Model-driven experimentation, Metabolic engineering

\footnotetext{
*Correspondence: zhangyun@im.ac.cn; wenty@im.ac.cn

${ }^{1}$ CAS Key Laboratory of Pathogenic Microbiology and Immunology,

Institute of Microbiology, Chinese Academy of Sciences, Beijing 100101,

China

${ }^{4}$ Savaid Medical School, University of Chinese Academy of Sciences,

Beijing 100049, China

Full list of author information is available at the end of the article
} 


\section{Background}

Corynebacterium glutamicum, a Gram-positive soil bacterium, is an important platform organism for industrial biotechnology and environmental remediation $[1,2]$. This microorganism is generally recognized as safe and is used in the fermentative production of L-glutamate and L-lysine on a million-ton scale and L-threonine, L-leucine and L-valine on a thousand-ton scale in the food, feed and pharmaceutical industries $[1,3]$. Due to the metabolic flexibility, broad substrate spectrum, and fermentative robustness of $C$. glutamicum cells, this bacterium has attracted attention as a potential cell factory to manufacture organic acids, bioplastic monomers (e.g., putrescine and cadaverine), and biofuels including ethanol, isobutanol, 1,2-propanediol, and 1-propanol [1, 4, 5]. Based on the knowledge of biochemical pathways and microbial fermentation processes, new technologies such as systems metabolic engineering and high-throughput analyses at the genome, transcriptome, proteome, and fluxome levels have been applied to strain development to improve the yield and productivity of desired products [6-10]. In silico genome-wide predictive simulation of metabolic profiles is expected to enable the design of artificial metabolic networks for desired product synthesis [11].

A genome-scale metabolic model (GEM) is constructed based on experimental data and information on gene annotations and functions, metabolites, reactions, enzymes, and their interactions [11, 12]. GEM has become an essential tool for understanding cellular metabolism, characterizing cell phenotypes, designing mutant strains with desired properties, and assessing the effects of genetic intervention and environmental perturbation on cellular metabolism [13]. The quality of a GEM determines the accuracy of outcomes for describing the genotype-phenotype relationship of a given strain $[13,14]$. The first GEM of Escherichia coli (iJE660) consisted of 660 genes, 627 reactions, and 438 metabolites. $i$ J6660 was constantly updated to $i \mathrm{JO} 1366$ by increasing the numbers of new genes and reactions, expanding the gene-protein-reaction associations (GPRs), balancing the reactions and compartmentalizing metabolites, among other updates [15-18]. The updated GEM accurately characterized $E$. coli growth metabolic profiles and was used to perform computational and quantitative analyses to resolve problems [14]. When coupled with metabolic flux constraints and a cellular objective, GEM analysis using computational algorithms [e.g., flux balance analysis (FBA), OptKnock, or OptForce] can predict the modification of targets in a metabolic network and successfully improve the production of succinic acid, ethanol, and malonyl-CoA in E. coli [19-21].

The initial GEM of C. glutamicum ATCC13032 (Model ${ }_{\mathrm{Cg}}$ 1) consisted of 247 genes, 446 reactions, and
411 metabolites and was updated to Model $_{\mathrm{Cg}} 2$ (277 genes, 502 reactions, and 423 metabolites) by revising inadequate reaction loops [22, 23]. Although these two models are useful, they have several limitations due to previously inadequate information. Annotated genes known to participate in different metabolic pathways, such as pyruvate kinase isoenzyme, glycerol-3-phosphatase, and (S,S)-butanediol dehydrogenase, were not included in Model $_{\mathrm{Cg}} 1$ or in Model $\mathrm{Cg}$ [24-26]. The deficiency of corresponding metabolic pathways in the two models prevented the prediction of cell growth on certain carbon sources (e.g., acetate, lactate, and xylose). The imbalance of the mass and charge in a few reactions (e.g., synthesis of RNA, protein, and biomass) and the directionality of certain reactions without thermodynamic constraints had a negative effect on genome-wide prediction using the two models. Moreover, the two models have incomplete L-glutamate metabolic systems, which prevented their application in studies of the metabolic state of L-glutamate production in the stationary phase [22, 27]. In 2016, Mei et al. constructed iJM658 based on the genome information of C. glutamicum S9114, which was generated by mutagenesis and is widely used for industrial L-glutamate production [27]. iJM658 contains L-glutamate secretion and uptake systems, which could be more suitable for studying L-glutamate production [27]. iJM658 is not applicable for the prediction of metabolic profile of C. glutamicum ATCC13032 due to the presence of numerous differences in the genome sequences between C. glutamicum S9114 and ATCC13032. Therefore, it is necessary to expand the scope of C. glutamicum ATCC13032' GEM to metabolic engineering for the desired products.

In this study, we present an updated model of the $C$. glutamicum ATCC13032 metabolic network reconstruction. This new model, named $i$ CW773, accurately predicted the growth ability and flux distributions of cells cultivated under different growth conditions. iCW773 simulations of genetic interventions for the overproduction of L-lysine, 1,2-propanediol, and isobutanol were highly consistent with experimental data. Along with wet-lab practices, genetic modifications were performed to generate a de novo L-proline-overproducing strain that exhibited the highest reported titer and productivity using a minimal medium in fed-batch fermentation.

\section{Results and discussion}

\section{A new genome-scale metabolic model of $C$. glutamicum ATCC13032}

We constructed a new GEM for ATCC13032, iCW773, which consisted of chemical reactions and interconverted metabolites. This network reconstruction contained 773 genes, 951 metabolites, and 1207 reactions, representing 
considerable increases in the numbers of genes, reactions and metabolites by 2.79-, 2.41-, and 2.25-fold, respectively, over Model $_{\mathrm{Cg}} 2$ (Additional file 1: Table S1). In the internal reactions of $i$ CW773, 709 ORFs $(89.18 \%)$ were extracted from the KEGG and UniProt databases [28]. The total ORF coverage was $25.75 \%$ in $i \mathrm{CW} 773$, which was $16.52 \%$ higher than that in Model $_{\mathrm{Cg}} 2$ (Additional file 1: Table S1). Five lumped reactions for fatty acids biosynthesis in $\operatorname{Model}_{\mathrm{Cg}}$ 2 were refined in $i$ CW773 by replacing each lumped reaction with several sequential reactions in $i$ CW773 (Additional file 2). Additionally, the charge and mass of reactions in $i$ CW773 were balanced, and the mass of reactions related to biomass was tested to be $1.00 \mathrm{~g} / \mathrm{g}$ dry cell weight (DCW) (Additional file 3). The directionality of reactions was constrained by the Gibbs free energy $\Delta_{r} G^{\prime}$, which was calculated using eQuilibrator ${ }^{2.0}$ [29]. A complete list of all the reactions and metabolites for $i \mathrm{CW} 773$ is provided in Additional file 3, and the central carbon metabolic pathway is presented in Additional file 4: Figure S1.

\section{Prediction of growth rates under different culture conditions}

To evaluate the quality of $i \mathrm{CW} 773$, the growth rates of $C$. glutamicum ATCC13032 cultivated on different carbon sources were predicted through FBA simulations using biomass maximization as the objective function. First, we calculated the growth rates on glucose using GEMs by setting the glucose and oxygen consumption rates as the experimental values [22, 30-32]. As shown in Fig. 1a, the Pearson's correlation coefficient (PCC) between the experimental and $i$ CW773-simulated data was consistent with that between the experimental and Model $_{\mathrm{Cg}} 2$-simulated data. When fructose, fructose mixed with glucose, sucrose, acetate, and lactate were set as the carbon sources, the cell growth rates on acetate or lactate could be calculated only by $i$ CW773, rather than Model $_{\mathrm{Cg}} 2$ (Fig. 1b) [33-37]. It has been reported that $C$. glutamicum was able to grow on non-native glycerol or xylose as the sole carbon source after expressing heterologous-related enzymes [38-40].

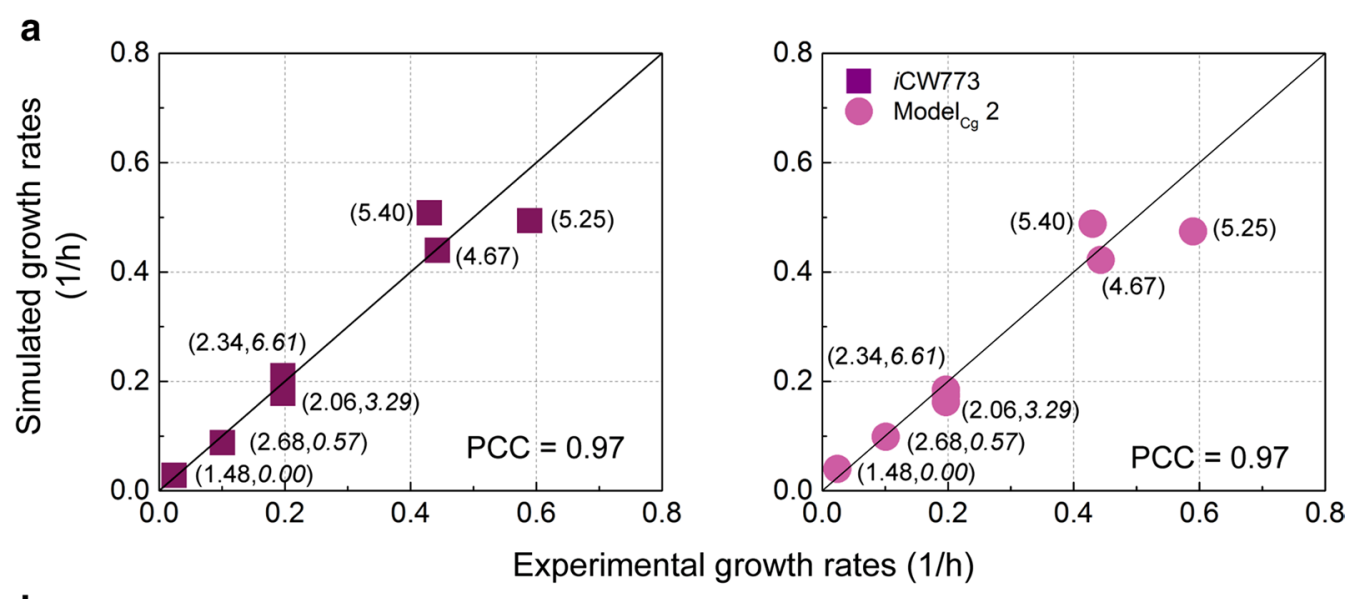

b
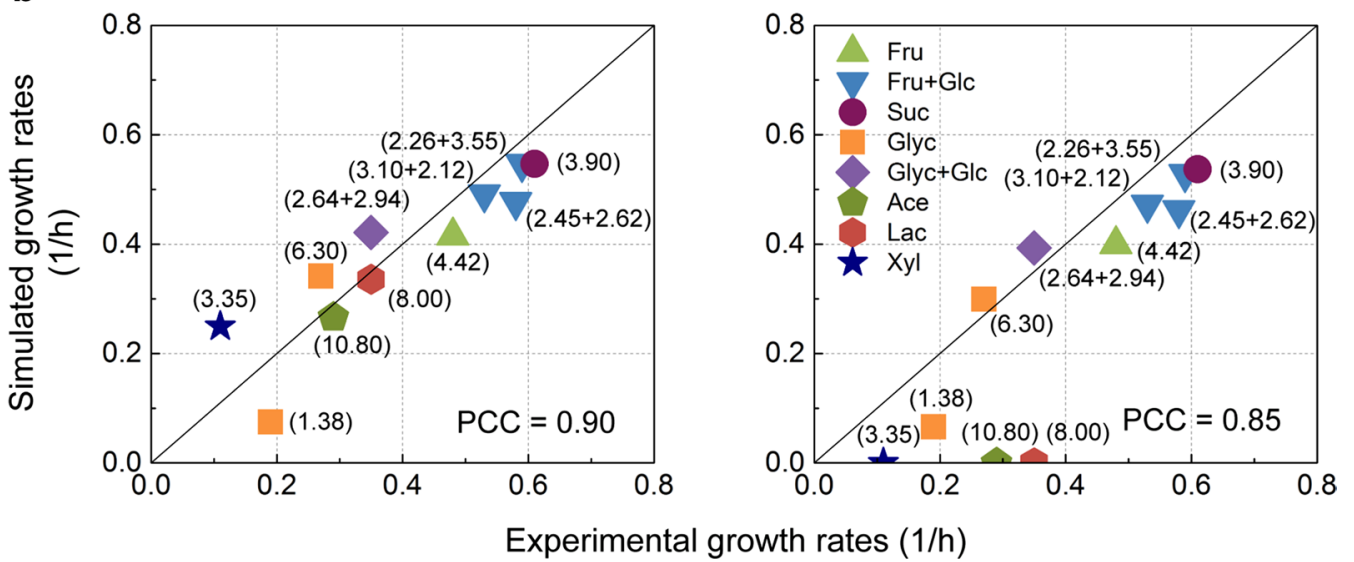

Fig. 1 Comparison of growth rates between experimental data and in silico simulation results at various glucose and oxygen supply levels (a) and different carbon sources (b) using iCW773 (left) and Model $_{\mathrm{Cg}} 2$ (right). PCC is the Pearson's correlation coefficient between the predicted product yield values and experimental data. The line corresponding to $x=y$ is also shown. $\mathbf{a}$ The glucose uptake rate is shown in regular font in parentheses, and the oxygen uptake rate is shown in italics in parentheses. $\mathbf{b}$ The uptake rate of the corresponding carbon source is shown in parentheses 
When the three reported reactions catalyzed by glycerol kinase, glycerol 3-phosphate dehydrogenase, and aquaglyceroporin from E. coli were added, both GEMs could calculate the biomass from glycerol [38, 39]. In contrast, only $i$ CW773 could simulate the biomass accumulation from xylose (Fig. 1b) when two reported reactions catalyzed by xylose isomerase and xylulokinase from $E$. coli were added [40]. These results provided evidence that supplemental reactions in $i \mathrm{CW} 773$ coupled the metabolism of the corresponding substrates (e.g., acetate, lactate, and xylose) with the central carbon metabolism (Additional file 5), resulting in the realization of growth simulations on these substrates.

\section{Metabolic profile prediction using iCW773}

Next, we compared model-predicted metabolic flux distributions to ${ }^{13} \mathrm{C}$-tracer metabolic flux data from ATCC13032 cultivated in minimal medium using glucose as the sole carbon source $[8,31,41]$. The biomass production was used as the objective function, and all flux data were normalized to the glucose uptake rate (100\%). Notably, the PCC between the experimental and $i$ CW773simulated fluxes was 0.99 , suggesting that $i C W 773$ provided an accurate intracellular flux distribution of the WT strain growing on glucose (Fig. 2a).

It has been reported that the inactivation of pgiencoding glucose-6-phosphate isomerase can lead to a significant metabolic flux disturbance, blocking the EMP pathway and redirecting the carbon flux from glucose-6-phosphate toward the PPP pathway in C. glutamicum [31]. To analyze the metabolic disturbance by gene knockout, the $p g i$ gene was deleted in $i$ CW773 and Model $_{\mathrm{Cg}}$ 2. When FBA was used to calculate the intracellular metabolic flux, the $i$ CW773-predicted flux toward the PPP pathway increased from 38.49 to $98.59 \%$,
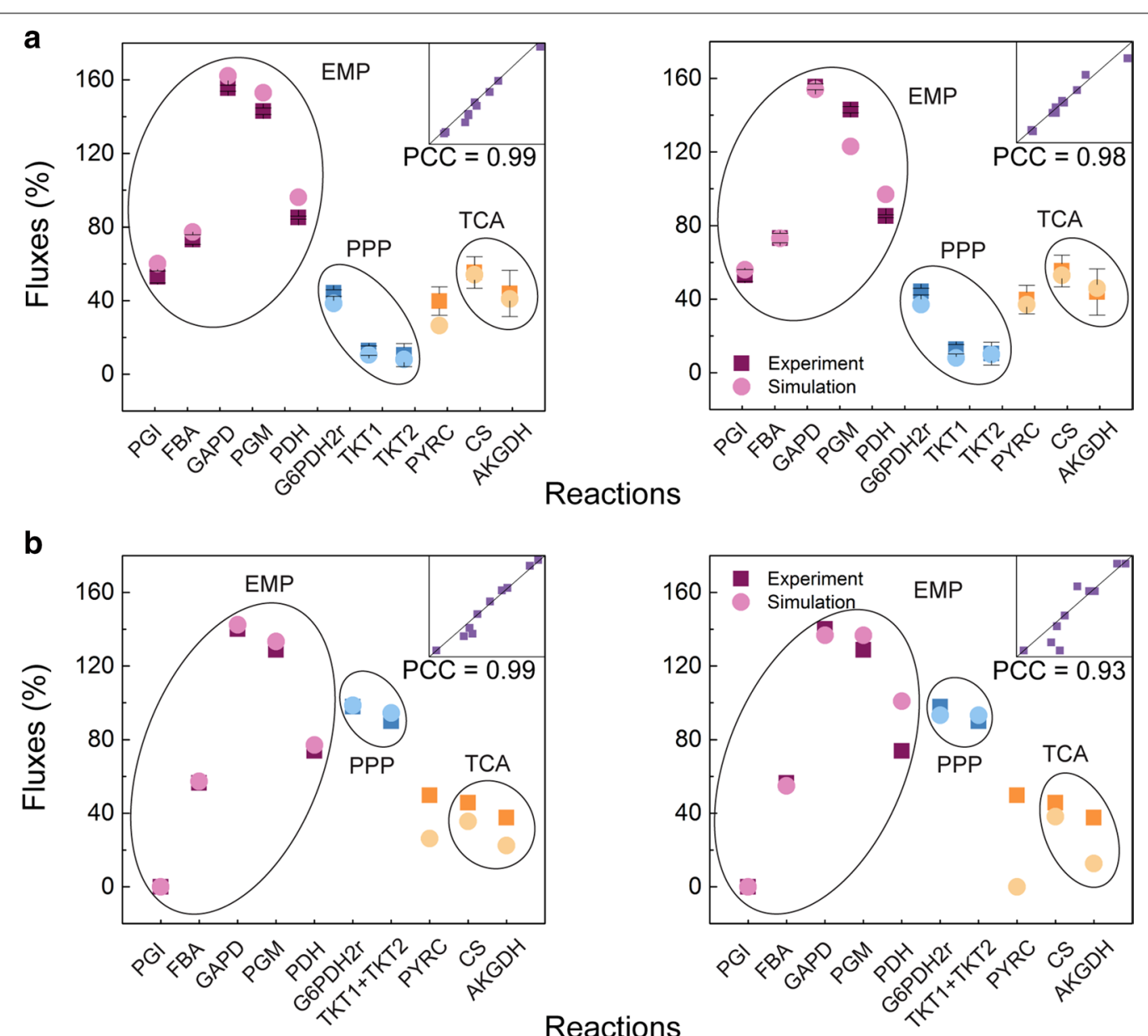

Reactions

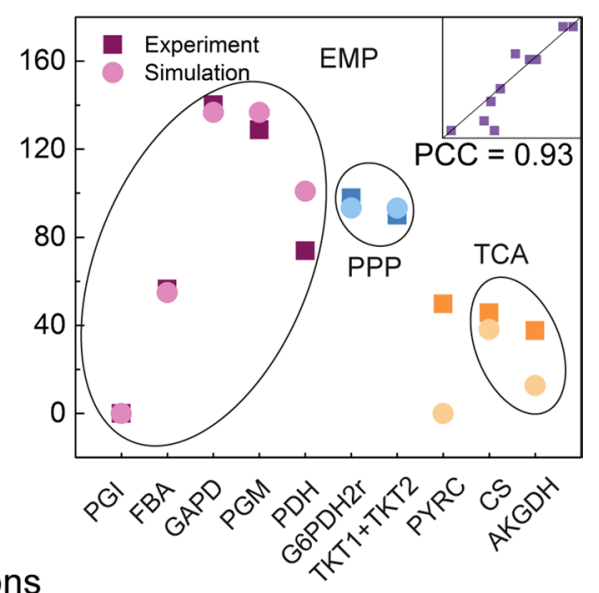

Fig. 2 Comparison of the metabolic flux profiles of the central metabolism between the ${ }^{13} \mathrm{C}$-tracer experimental WT (a) and WT $\triangle p$ gi (b) data for the exponential growth phase and in silico simulation results using iCW773 (left) and Model $_{\mathrm{Cg}} 2$ (right). Fluxes were normalized to the glucose uptake rates, which were set to 100. PCC is the Pearson's correlation coefficient between the predicted product yield values and experimental data. The line corresponding to $x=y$ is also shown in the inserted figure in the top right corner. EMP: Embden-Meyerhof pathway (glycolytic pathway); PPP: pentose phosphate pathway; TCA: tricarboxylic acid cycle. a The experimental data were derived from three studies, and the error bars represent the standard deviations $[8,31,41]$ 
which was consistent with the experimental values (from 44.10 to $97.80 \%$ ) (Fig. 2b) [31]. Moreover, the simulated amount of NADPH generated from the PPP pathway in WT $\Delta p g i$ was $1.97 \mathrm{~mol} / \mathrm{mol}$ glucose, which was 2.30 -fold higher than that generated from the PPP pathway in WT $(0.77 \mathrm{~mol} / \mathrm{mol}$ glucose). Due to the requirement for a certain amount of NADPH to generate biomass in the simulation (e.g., $4 \mathrm{~mol} \mathrm{NADPH}$ per mol L-lysine and $2 \mathrm{~mol}$ $\mathrm{NADPH}$ per mol L-valine), the NADPH generated from isocitrate dehydrogenase in the TCA cycle decreased from $0.41 \mathrm{~mol} / \mathrm{mol}$ glucose to $0.22 \mathrm{~mol} / \mathrm{mol}$ glucose to maintain a stoichiometric balance [42, 43]. This difference in NADPH generation resulted in a smaller deviation from the $i$ CW773-simulated TCA flux to the experimental value, as shown in Fig. 2b (left). Notably, the flux of pyruvate carboxylase (PYRC) in $i$ CW773 was efficiently generated, while no PYRC flux from pyruvate to oxaloacetate was calculated in $\operatorname{Model}_{\mathrm{Cg}} 2$. These results illustrate the suitability of $i \mathrm{CW} 773$ for predicting intracellular metabolic flux distribution.

\section{Validation of iCW773 simulation for amino acid overproduction}

To investigate whether $i \mathrm{CW} 773$ is an efficient tool to design strategies for the metabolic engineering of C. glutamicum, the ranges of flux variability in the network for the WT and L-lysine overproducing strains were calculated using OptForce ${ }_{\text {MUST }}$ (Additional file 6). As shown in Fig. 3, the gap between the flux ranges of the WT and overproducing network quantified the degree of required reaction flux modification. In the simulations of $i$ CW773, the upregulation of aspartokinase (encoded by $l y s C$ ), dihydrodipicolinate synthase (encoded by $\operatorname{dap} A$ ), dihydrodipicolinate reductase (encoded by dapB), diaminopimelate dehydrogenase (encoded by $d d h$ ), and diaminopimelate decarboxylase (encoded by $l y s A$ ) in the L-lysine synthesis pathway were consistent with previous reports $[8,44,45]$. Downregulation of homoserine dehydrogenase (encoded by hom) decreased the flux toward the competitive pathway $[8,44,46]$. Moreover, deletion of phosphoenolpyruvate carboxykinase (encoded by $p c k$ ) and upregulation of pyruvate carboxylase (encoded by pyc) directed the flux toward oxaloacetate formation at the pyruvate node for L-lysine synthesis [8, 47]. Downregulation of aconitase (encoded by $a c n$ ) and isocitrate dehydrogenase (encoded by $i c d$ ) further minimized carbon loss from pyruvate [48]. In addition, upregulation of the entire transketolase operon and fructose-1,6-bisphosphatase (encoded by $f b p$ ) enhances the NADPH supply via PPP to increase L-lysine production $[8,49]$. In contrast, Model $_{\mathrm{Cg}} 2$ failed to predict the deletion of $p c k$, the downregulation of hom and the upregulation of $l y s C, d d h, d a p B$, and $f b p$. Therefore, the predictions by $i$ CW773 were highly consistent with experimental results. For L-valine and L-serine overproduction, the modification targets predicted by $i$ CW773 were identical to the reported experimental results (Additional file 1: Table S2).

\section{Validation of iCW773 simulation for non-native 1,2-propanediol and isobutanol synthesis}

Corynebacterium glutamicum has previously been engineered for non-native 1,2-propanediol and isobutanol production [50, 51]. Therefore, we performed a comparison between $i \mathrm{CW} 773$-simulated and experimentally performed genetic modification strategies with respect to 1,2-propanediol and isobutanol overproduction [50, 51]. As previously reported, a three-reaction synthesis route by the heterologous genes $m g s A$, gldA, and $y q h D$, which encode methylglyoxal synthase, glycerol dehydrogenase, and alcohol dehydrogenase from $E$. coli, was added to $i$ CW773 to synthesize 1,2-propanediol from dihydroxyacetone phosphate (dhap) [50]. Then, the ranges of flux variability in the network for the WT and 1,2-propanediol-overproducing strains were calculated by OptForce $_{\text {MUST }}$ (Additional file 7). As shown in Fig. 4a, upregulation of $m g s A, g l d A$, and $y q h D$ directed the flux toward 1,2-propanediol synthesis, which was consistent with the finding that the heterologous expression of these three genes in the WT strain led to a product yield of $0.13 \mathrm{~mol} / \mathrm{mol}$ (1,2-propanediol/glucose) [50]. Moreover, model simulation showed that the deletion of $h d p A$ encoding dihydroxyacetone phosphate phosphatase could increase the precursor (dhap) supply by preventing glycerol formation from dhap, which was supported by the experimental results that 1,2-propanediol production increased by $90.08 \%$ in strain WT $\Delta h d p A$ (pEKEx3-mgsAgldA-yqhD) [50]. The OptForce ${ }_{\text {MUST }}$ analysis indicated that knockout of $l d h$ encoding lactate dehydrogenase could further decrease the formation of byproducts and increase 1,2-propanediol production. This prediction was validated by the experimental finding that $l d h$ deletion increased 1,2-propanediol by $37.75 \%$ [50]. In addition, downregulation of tpiA, gap, $p g k$, and $g p m A$ would be novel targets for 1,2-propanediol overproduction.

As reported previously, the isobutanol pathway in $i$ CW773 was constructed by kivd-encoding keto acid decarboxylase from Lactococcus lactis and native adhAencoding alcohol dehydrogenase catalyzing 3-methyl2-oxobutanoate to isobutanol [52]. The predicted knockout, upregulation, and downregulation of target genes by OptForce ${ }_{\text {MUST }}$ are shown in Fig. 4b (Additional file 7). The simulation was consistent with the experimental results that the upregulation of four genes (alsS, $i l v C D$, kivd, and adhA) could endow C. glutamicum with isobutanol synthesis capability [52]. The OptForce MUST $_{\text {T }}$ 


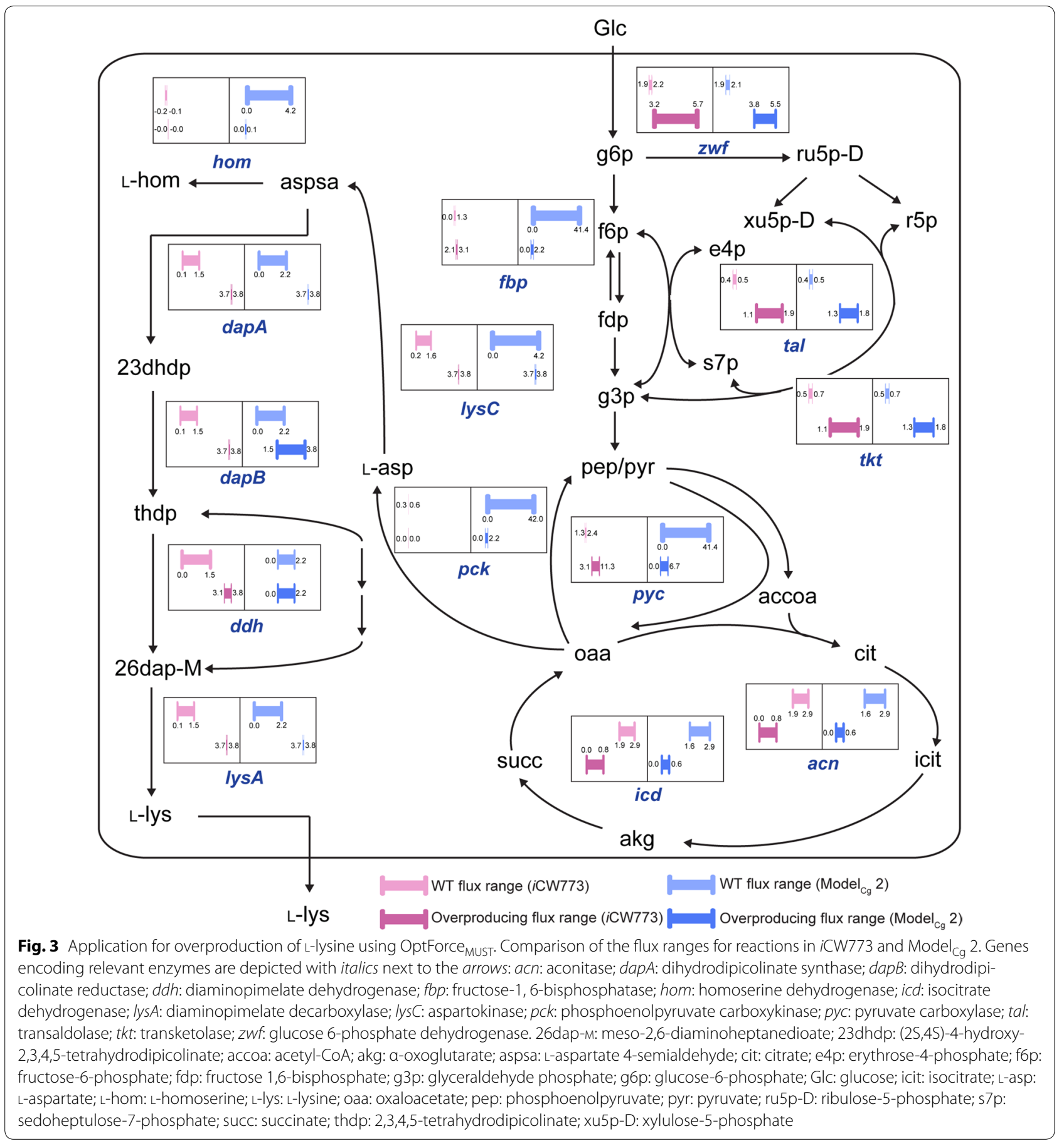

analysis indicated that the decrease in pyruvate consumption caused by inactivating ldh-encoding lactate dehydrogenase and downregulating the aceE-encoding pyruvate dehydrogenase subunit would increase isobutanol synthesis. In vivo $l d h$ deletion increased the isobutanol yield by $23 \%$, while deletion of $a c e E$ led to pyruvate overflow to lactate formation rather than increased isobutanol synthesis [52]. It was reported that aceE deletion caused C. glutamicum to be unable to grow with glucose unless supplemented with acetate, indicating that knockout of aceE has a negative effect on cell growth and that knockdown of aceE, as suggested by the $i C W 773$ 


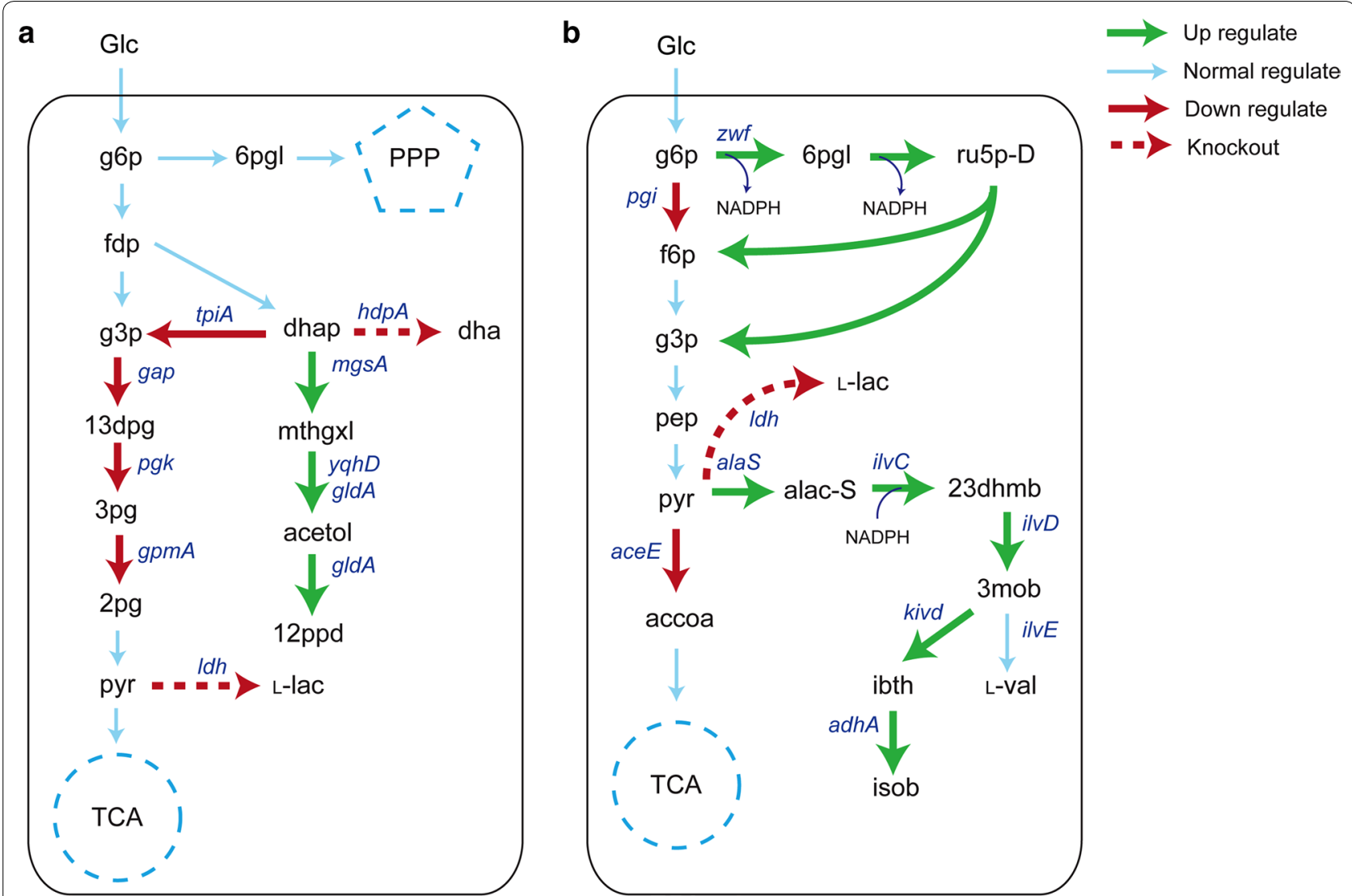

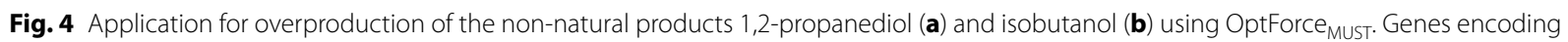
relevant enzymes are depicted with italics next to the arrows: aceE: pyruvate dehydrogenase complex; adhA: alcohol dehydrogenase; alaS: acetolactate synthase; gap: glyceraldehyde-3-phosphate dehydrogenase; gldA: glycerol dehydrogenase; gpmA: phosphoglycerate mutase; $h d p A$ : dihydroxyacetone phosphate phosphatase; ilvC: acetohydroxyacid isomeroreductase; ilvD: dihydroxyacid dehydratase; ilvE: valine transaminase; kivD: keto acid decarboxylase; Idh: lactate dehydrogenase; mgsA: methylglyoxal synthase; pgi: glucose-6-phosphate isomerase; pgk: phosphoglycerate kinase; tpiA: triose-phosphate isomerase; yqhD: aldehyde reductase. For other genes, see Fig. 3. 12ppd: 1,2-propanediol; 13dpg: 3-phospho-D-glyceroyl phosphate; 23dhmp: 2,3-dihydroxy-3-methylbutanoate; 2pg: D-glycerate 2-phosphate; 3mob: 3-methyl-2-oxobutanoate; 3pg: 3-phospho-D-glycerate; 6pgl: 6-phospho-D-glucono-1,5-lactone; acetol: acetol; alas-S: 2-acetolactate; dha: dihydroxyacetone; dhap: dihydroxyacetone phosphate; ibth: isobutyraldehyde; isob: isobutanol; L-lac: L-lactate; mthgxl: methylglyoxal; L-val: L-valine. For other abbreviations, see Fig. 3

simulation, might be a better alternative [53]. In addition, the $i$ CW773-simulated strategy to improve isobutanol synthesis included an increase in the intracellular NADPH pool by downregulation of $p g i$ in combination with upregulation of $z w f$. This combined regulation mode for $p g i$ and $z w f$ had been demonstrated to increase intracellular NADPH levels by redirecting carbon flux toward PPP [9]. In contrast, deletion of pgi together with aceE severely inhibited growth and eliminated isobutanol production [52]. Taken together, these data indicate that downregulation of aceE and $p g i$ together with upregulation of $z w f$ would contribute to isobutanol synthesis and simultaneously maintain growth. In summary, the $i$ CW773-designed strategy could provide insights into future work for the optimization of 1,2-propanediol and isobutanol production in C. glutamicum.

\section{In silico design and wet-lab construction of an L-proline-overproducing strain}

Despite the strong flux toward L-glutamate formation in C. glutamicum, L-proline converted from L-glutamate under three sequential catalytic reactions by $\gamma$-glutamyl kinase (proB), glutamate-5-semialdehyde dehydrogenase (proA), and pyrroline-5-carboxylate reductase (proC) has not been efficiently synthesized by the engineered strain derived from C. glutamicum ATCC13032 [54-56]. The heterologous expression of ornithine cyclodeaminase merely made an L-ornithine-producing strain overproduce L-proline by six enzymatic reactions from L-glutamate, which was not an economically feasible means of carbon utilization in L-proline synthesis [56, 57]. Thus, $i$ CW773 was applied to identify metabolic interventions that led to the overproduction of 
L-proline via OptForce ${ }_{\text {MUST }}$ (Additional file 8). As shown in Fig. 5a, the upregulation of five reactions in the glycolytic pathway and citrate synthase (CS, encoded by gltA) and downregulation of $\alpha$-oxoglutarate dehydrogenase (AKGDH, encoded by $k g d$ ) predicted by $i$ CW773 were consistent with those predicted by $\operatorname{Model}_{\mathrm{Cg}} 2$. The differences between the $i \mathrm{CW} 773$ and Model $_{\mathrm{Cg}} 2$ simulations were the downregulation of reactions from pyruvate to L-valine and L-alanine and upregulation of aconitase [ACONTa(b), encoded by $a c n$ ] and isocitrate dehydrogenase (ICDHyr, encoded by icd), which converts citrate to $\alpha$-oxoglutarate. The largest difference between the two models was the knockout of the putA gene to block the conversion of $\Delta 1$-pyrroline-5-carboxylate to glutamate. PutA is a bifunctional enzyme with both proline dehydrogenase and $\Delta 1$-pyrroline-5-carboxylate dehydrogenase activities, which catalyzes the two-step oxidation of L-proline to glutamate in E. coli [58]. In $i$ CW773, the reaction from $\mathrm{L}$-glutamate to $\Delta 1$-pyrroline-5-carboxylate formed a cycle in the presence of PutA, which made it inefficient to convert $\Delta 1$-pyrroline-5-carboxylate to L-proline. Blocking this conversion and upregulation of
$\Delta 1$-pyrroline-5-carboxylate reductase (encoded by proC), the flux could be efficiently directed toward L-proline synthesis. Then, the PutA reactions were deleted in both models to predict the metabolic profile of overproducing L-proline (Additional file 9). As shown in Fig. 5b, one difference between the $i \mathrm{CW} 773$ and $\operatorname{Model}_{\mathrm{Cg}} 2$ simulations was the upregulation of ProB to direct the metabolic flux from L-glutamate toward L-proline synthesis. However, the reaction from glutamate-5-semialdehyde to $\Delta 1$-pyrroline-5-carboxylate, which differed in the Model $_{\mathrm{Cg}} 2$ simulation, was impossible to control by genetic modification because this reaction occurred spontaneously without enzymatic catalysis.

To validate the accuracy of the $i \mathrm{CW} 773$ prediction for L-proline overproduction, different targets between the $i \mathrm{CW} 773$ and Model $_{\mathrm{Cg}} 2$ simulations were chosen for genetic modification. Because the enzymatic activity of ProB is strictly restricted to feedback inhibition by L-proline, no accumulation of L-proline will occur in WT [59]. Therefore, the feedback inhibition of ProB by L-proline was first released through the introduction of the nucleotide substitution G446A into the chromosomal proB

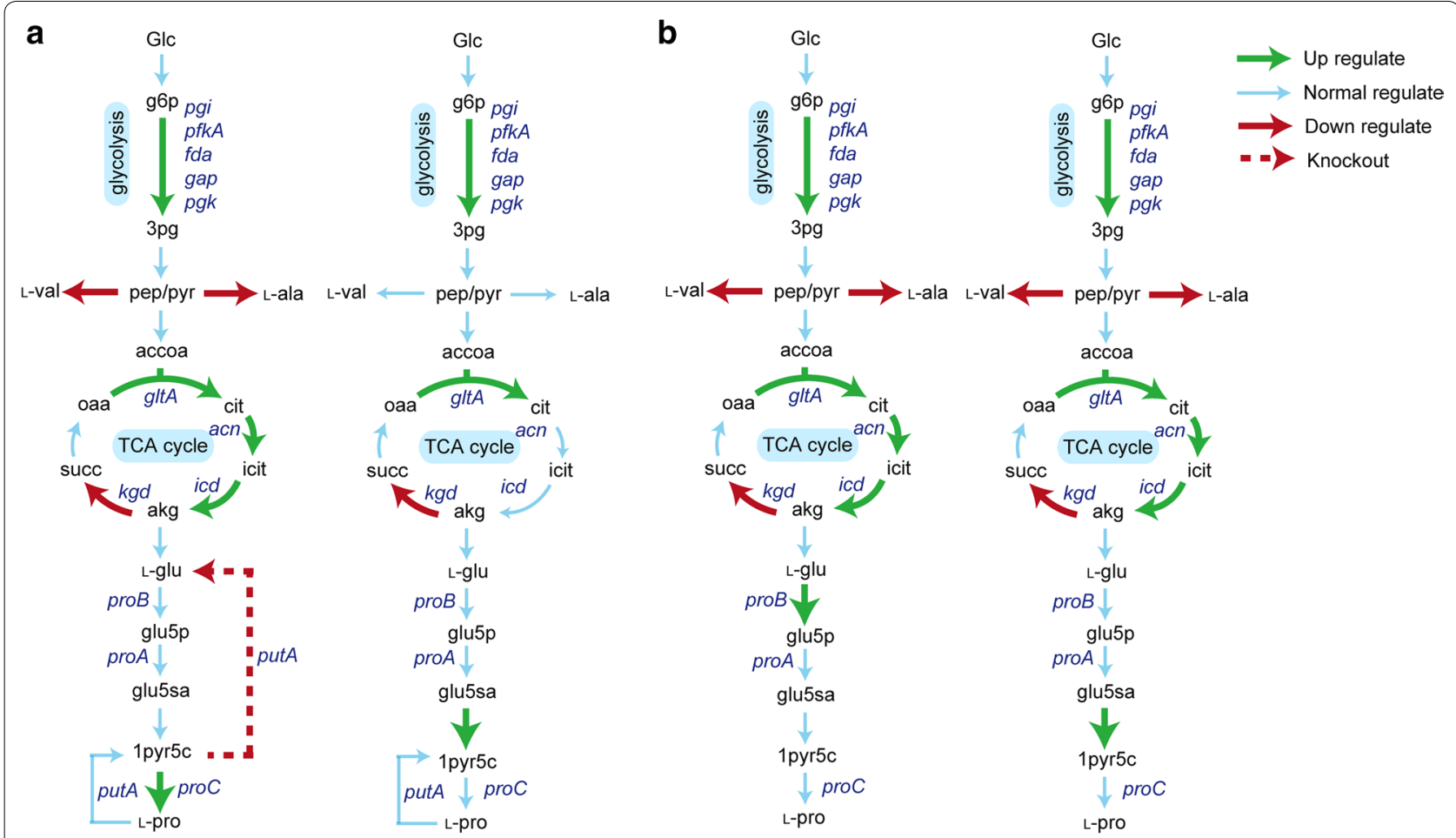

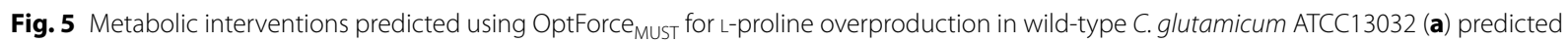
by iCW773 (left) and Model $\mathrm{Cg}_{2}$ (right) and in the mutant strain C. glutamicum ATCC13032 $\triangle$ putA (b) predicted by iCW773 (left) and Model 2 (right). Genes encoding relevant enzymes are depicted in italics next to the arrows: fda: fructose-bisphosphate aldolase; gltA: citrate synthase; kgd: a-oxoglutarate dehydrogenase; pfkA: phosphofructokinase; proA: glutamate-5-semialdehyde dehydrogenase; proB: $\gamma$-glutamyl kinase; proC: pyrroline-5-carboxylate reductase; putA: proline dehydrogenase and $\triangle 1$-pyrroline-5-carboxylate dehydrogenase. For other genes, see Figs. 3 and 4. 1 pyr5c: $\Delta$-pyrroline-5-carboxylate; glu5p: glutamate-5-phosphate; glu5sa: glutamate-5-semialdehyde; L-ala: L-alanine; L-glu: L-glutamate; L-pro: L-proline. For other abbreviations, see Figs. 3 and 4 
gene [60]. The Pro-1 strain accumulated a small amount of L-proline compared with the WT (Table 1). Notably, the L-proline titer dropped after 36 h (Fig. 6a), indicating that L-proline may be converted to L-glutamate under PutA catalysis as simulated by $i$ CW773. When putA was knocked out in Pro-1, the L-proline production of Pro-2 was 1.92-fold higher than that of Pro-1 (Table 1), which was consistent with a previous report that deletion of putA significantly increased the formation of trans-4-Lhydroxyproline derived from L-proline in recombinant $E$. coli BL21 [61]. In addition, the product yield of L-proline from glucose was improved 1.94-fold upon deleting putA (Fig. 6a).

Upregulation of $g l t A$, acn, and icd expression was simulated by $i$ CW773 to drive more carbon flux to $\alpha$-oxoglutarate formation for L-proline synthesis. It has been demonstrated that CS flux in vivo is not strictly controlled by the amount of gltA expression [62], while the transcription of the $a c n$ gene is subjected to complex regulations mediated by the AcnR, RipA, and GlxR repressors $[63,64]$. Thus, aconitase-catalyzed reactions should be a major control point in the TCA cycle during growth on glucose $[64,65]$. The effects of increased $\mathrm{ACONTa}(\mathrm{b})$ flux on cell metabolism and L-proline synthesis were simulated using FBA. The fluxes toward CS and ICDHyr were enhanced with the increase in the relative $\mathrm{ACONTa}(\mathrm{b})$ flux, and maximum extracellular L-proline production was achieved when the relative ACONTa(b) flux was improved by 2.5 -fold compared with WT (Fig. 6b). Conversely, the fluxes toward L-valine and L-alanine synthesis decreased. After the native promoter of the $a c n$ gene was replaced with three promoters of varying strength $\left(\mathrm{P}_{g l y A}, \mathrm{P}_{p c k}\right.$, and $\left.\mathrm{P}_{e f t u}\right)$ and the start codon was changed from TTG to ATG in Pro-2, the mRNA levels of the acn gene increased by 1.47-, 1.82-, and 2.83-fold in the Pro-3, Pro-4 and Pro-5 strains, respectively (Fig. 6c). With upregulation of the acn gene, the mRNA levels of $g l t A$ in Pro- 4 and Pro- 5 increased by 50.82 and $88.52 \%$, respectively, consistent with a previous observation that the expression of $g l t A$ and acn were simultaneously increased and might be controlled by a similar mechanism [65]. However, no significant changes were observed in the mRNA level of icd or kgd (Fig. 6c). When the mRNA levels of $a c n$ were increased, Pro-5 produced $2.26 \pm 0.03 \mathrm{~g} / \mathrm{L}$ L-proline with a $33.73 \%$ increase over Pro-2. Correspondingly, the byproducts of $\mathrm{L}$-alanine and L-valine decreased by 16.95 and $8.09 \%$. The extracellular metabolites exhibited a $73.34 \%$ decrease in citrate and a 1.37 -fold increase in $\alpha$-oxoglutarate.

Finally, to validate the positive correlation between GLU5K flux and L-proline yields (Additional file 4: Figure S2), the mutant proB (G446A) under the control of $\mathrm{P}_{t a c}$ on a plasmid was induced to overexpress the feedback-resistant $\gamma$-glutamyl kinase. When the mRNA level of rro $^{*}$ was significantly increased, the mRNA levels of the proA, proC, and $g d h$ genes were simultaneously increased (Fig. 6c). The resultant Pro-6 strain showed an 8.27 -fold increase in L-proline production $(18.71 \pm 0.21 \mathrm{~g} / \mathrm{L})$, with 8.18 - and 9.03-fold increases in yield $(0.36 \mathrm{~g} / \mathrm{g})$ and productivity $(0.32 \mathrm{~g} / \mathrm{L} / \mathrm{h})$, respectively, compared with Pro-5 (Fig. 6a). In addition, there were 85.55 and $45.42 \%$ decreases in extracellular $\alpha$-oxoglutarate and succinate, respectively.

\section{The performance of Pro- 6 in fed-batch fermentation}

The production performance of the final Pro- 6 strain was investigated in fed-batch fermentation. As shown in Fig. 7, the strain grew continuously from 0 to $32 \mathrm{~h}$ and reached a cell concentration of $\mathrm{OD}_{600}=109.02$ at $32 \mathrm{~h}$. The assimilated glucose was efficiently channeled to the L-proline biosynthetic pathway; L-proline production began at an early stage and continuously increased throughout the fermentation period. A major and constant increase in L-proline production was achieved during the feeding phase, which was initiated after the initial sugar $(40 \mathrm{~g} / \mathrm{L})$ in the batch medium was consumed. The maximal specific growth rate of strain Pro-6 was $0.40 \mathrm{~h}^{-1}$, and the maximal specific glucose consumption rate was $5.46 \mathrm{mmol} / \mathrm{gDCW} / \mathrm{h}$. The maximal L-proline titer reached $66.43 \mathrm{~g} / \mathrm{L}$ at $60 \mathrm{~h}$ with a yield of $0.26 \mathrm{~g} / \mathrm{g}$ glucose $(0.41 \mathrm{~mol} / \mathrm{mol}$ glucose $)$ and a productivity of $1.11 \mathrm{~g} / \mathrm{L} / \mathrm{h}$. Consequently, the genetically engineered Pro- 6 strain in this study was demonstrated to be an efficient L-proline-producing strain without any nutrition-auxotroph and with the highest titer and productivity reported on minimal medium with glucose as the sole carbon source (Table 2).

\section{Conclusions}

In this study, we reconstructed a genome-scale metabolic model of C. glutamicum ATCC13032 with an expanded size and scope compared with the previous $\operatorname{Model}_{\mathrm{Cg}} 2$. The $i$ CW773-simulated results of growth rates, metabolic flux profiles, and modification targets for overproducing natural and non-natural products were much more consistent with experimental data than those of $\operatorname{Model}_{\mathrm{Cg}} 2$. Moreover, in silico analysis of L-proline overproduction and modifications of only three genes (putA, acn, and proB) cooperatively forced carbon flux toward L-proline, with the highest titer and productivity reported on minimal medium. This work revealed that the genome-scale metabolic model successfully guided the wet-lab experiments. This development will further boost C. glutamicum, one of the most promising and valuable workhorses, into a new era of industrial microbial biotechnology beyond the classical field. 


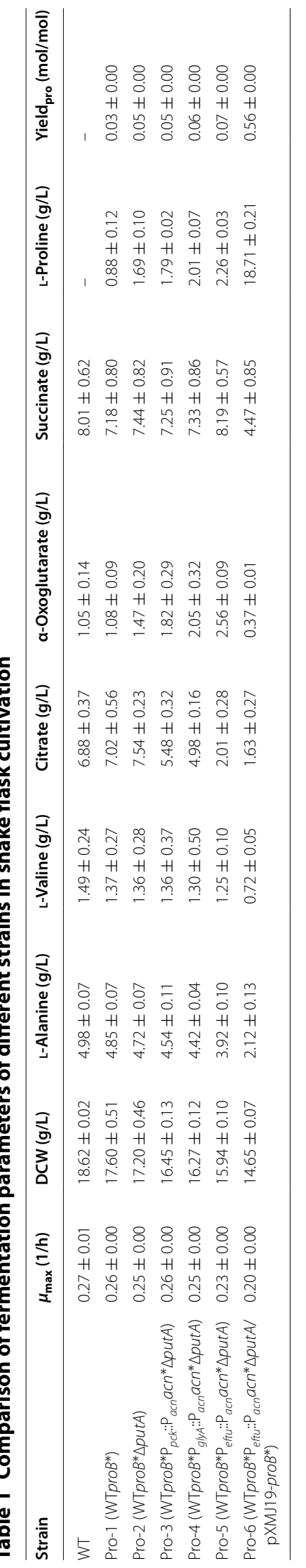




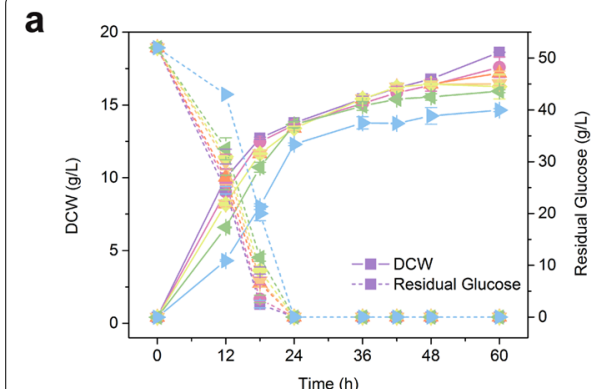

b

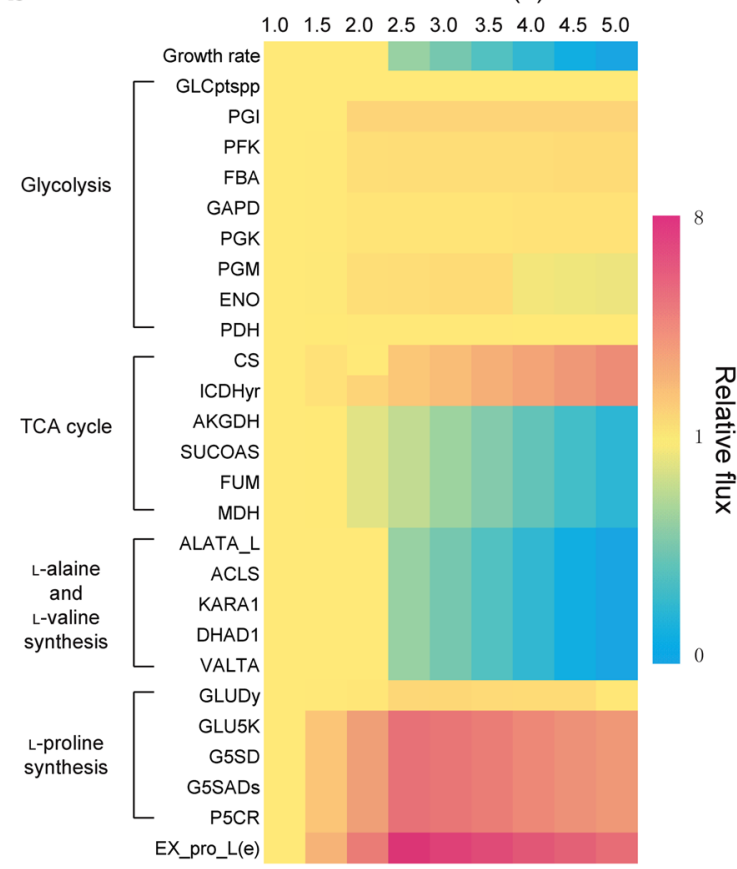

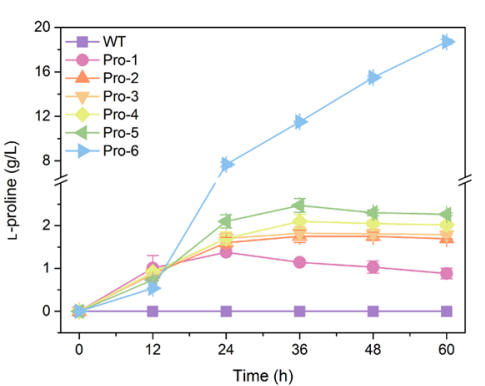

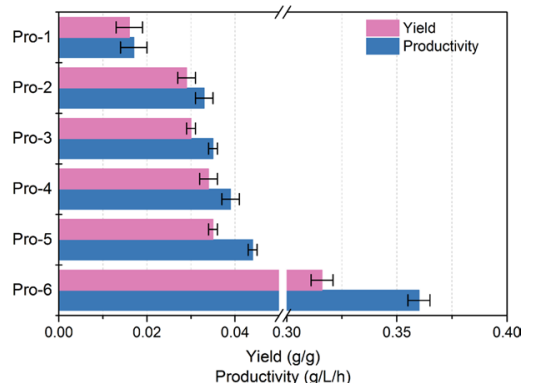

C
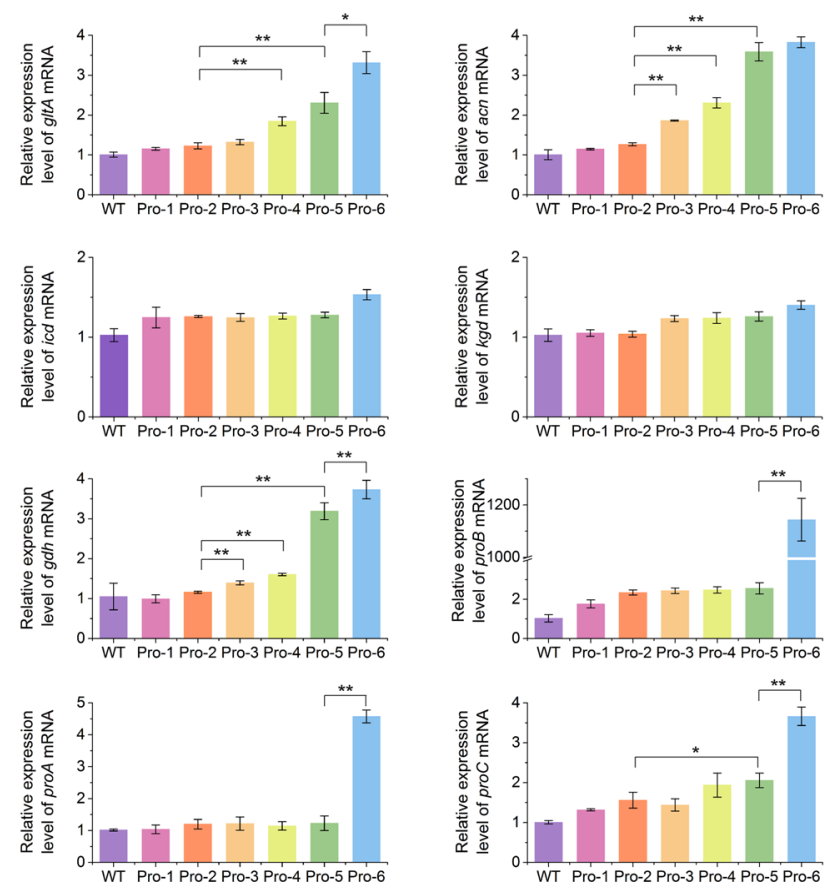

Fig. 6 Analysis of L-proline-overproducing strains. a Flask cultivation of different C. glutamicum strains. $\mathbf{b}$ Simulation of the impact of increased ACONTa(b) flux on cellular metabolism for robustness analysis was investigated with increasing relative ACONTa(b) fluxes (between 1.0 and 5.0). $X$-axis relative $\mathrm{ACONTa}(\mathrm{b})$ flux (the ratio of the test $\mathrm{ACONTa}(\mathrm{b})$ flux to the native $\mathrm{ACONTa}(\mathrm{b})$ flux). Relative $\mathrm{ACONTa}(\mathrm{b})$ flux $=1.0$ served as the control (or native GPD flux). Y-axis relative flux of the pathways (the ratio of the test flux to the native flux). Yellow represents no change, red represents upregulation, and blue represents downregulation of the flux. Descriptions of abbreviated reaction names are provided in Additional file 3. c Relative transcript expression of related genes of the analyzed C. glutamicum strains in the exponential growth phase in glucose. Significant differences in the data were determined using Student's $t$ test $\left({ }^{*} P<0.05,{ }^{* *} P<0.01\right)$. The data were derived from experiments performed at least three times, and the error bars represent the standard deviations

\section{Methods}

\section{Genome-scale metabolic model reconstruction}

The reconstruction of the C. glutamicum ATCC13032 metabolic network was conducted based on the model of Shinfuku et al. [22]. A 96-step procedure for metabolic network reconstruction was recently published [66], and the appropriate steps were followed after new genes, reactions, and metabolites were added to generate $i \mathrm{CW} 773$. The KEGG (http://www.genome.jp/kegg/) and UniProt (http:// www.uniprot.org/) databases were referenced to obtain reaction information and reaction-gene associations during the draft reconstruction stage, and $i \mathrm{JO} 1366$ was referenced to obtain the abbreviations of the same metabolites between C. glutamicum and E. coli [17]. The charge and mass of reactions in $i$ CW773 were automatically balanced using the CheckMassChargeBalance command in Cobra Toolbox, and reversibility was assigned based on the Gibbs free energy by the eQuilibrator database (http:// equilibrator2.milolab.webfactional.com/). Cobra Toolbox 2.05 with MatLab 2010b (MathWorks Inc.) was used for additional model testing [67], and the glpk solvers were used for the optimization procedures [68]. 


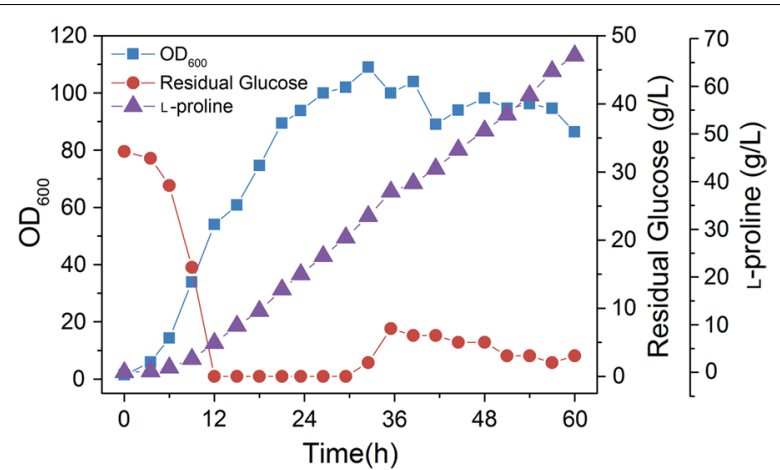

Fig. 7 Fed-batch culture of Pro-6 in a 7.5-L bioreactor

\section{Flux balance analysis}

The growth rates and metabolic flux distribution of $C$. glutamicum ATCC13032 were calculated using FBA with commercially available glpk and Matlab software $[16,18]$. To simulate growth rates and metabolic flux distributions, biomass production was set as the objective function. For the simulation of growth rates under different glucose and oxygen levels, glucose and oxygen uptake rates were set as the experimental values, as shown in Fig. 1a. For the simulation of growth rates on different carbon sources, the uptake rate of related carbon source was set as the experimental value, as shown in Fig. 1b. Small molecular external metabolites such as $\mathrm{CO}_{2}, \mathrm{H}_{2} \mathrm{O}$, $\mathrm{SO}_{3}, \mathrm{NH}_{3}$, and $\mathrm{PO}_{4}$ were allowed to be freely transported across the cell membrane.

\section{OptForce $_{\text {MUST }}$}

The flux variability analysis was conducted to calculate each reaction's flux span for both the wild-type and overproducing strains. $\mathrm{A}{ }^{13} \mathrm{C}$ flux and a relatively high secretion rate analyses were employed to constraint the wild-type and overproducing-type simulations, respectively [69]. The flux ranges for each reaction were compared, and some reactions of the overproducing strain need to be genetically modified through up/downregulation or knockout if no overlap between the flux range of the wild-type strain and that of the overproducing strain was found. A pool of modified reactions to construct the overproducing-type strain was produced.

To analyze the overproductions of the native and nonnative metabolites, ${ }^{13} \mathrm{C}$ flux data of the wild-type strain on glucose under aerobic condition were used as the constraints of the maximal range of flux variability $[8,31,41]$. For the non-native product, simulations of 1,2-propanediol and isobutanol production were performed after the heterogeneous reactions were added to $i \mathrm{CW} 773$, as described in Additional file 10. We imposed a minimum production yield of $98 \%$ of the theoretical maximum for L-lysine, L-valine, L-serine, L-proline, 1,2-propanediol, and isobutanol; the biomass flux was constrained to at least $1 \%$ of its theoretical maximum; and the L-lysine, L-valine, L-serine, L-proline, 1,2-propanediol, and isobutanol transport reactions were set as the target reactions, respectively. The upper and lower bounds of the ${ }^{13} \mathrm{C}$-tracer experimental fluxes are listed in Additional file $10[8,31,41]$. OptForce MUST $_{\text {was }}$ used to identify the reactions/genes that required to be up/downregulated or knocked out to maximize the production of the targeted metabolites.

\section{Strains and plasmids}

Wild-type C. glutamicum ATCC13032 (American Type and Culture Collection, Manassas, VA, USA) was used as the parental strain for strain engineering. The $E$. coli strain EC135 was used as the cloning host, and the pXMJ19 and pK18mobsacB plasmids were applied for gene overexpression with induction of isopropyl- $\beta$-Dthiogalactopyranoside (IPTG) and gene deletion, respectively [70]. All of the strains and plasmids used in this study are listed in Additional file 1: Table S3.

\section{Constructions of plasmids and strains}

Total genomic DNA was extracted from C. glutamicum according to a previously described procedure [71].

Table 2 Overview of L-proline-producing strains

\begin{tabular}{|c|c|c|c|c|c|c|}
\hline Strain & Breeding & Medium & Titer (g/L) & Yield (g/g) & Productivity (g/L/h) & References \\
\hline \multirow[t]{2}{*}{ Pro-6 } & Metabolic engineering & Minimal medium & $18.71 \pm 0.21$ & $0.36 \pm 0.01$ & $0.32 \pm 0.01$ & This work \\
\hline & & & $66.43^{b}$ & $0.26^{\mathrm{b}}$ & $1.11^{\mathrm{b}}$ & \\
\hline Corynebacterium glutamicum & Metabolic engineering & Minimal medium & & $0.25-0.30^{a}$ & $0.25-0.30^{\mathrm{a}}$ & {$[57]$} \\
\hline Corynebacterium glutamicum & Metabolic engineering & Minimal medium & 12.70 & 0.36 & 0.42 & {$[56]$} \\
\hline $\begin{array}{l}\text { Corynebacterium acetoacido- } \\
\text { philum }\end{array}$ & $\begin{array}{l}\text { L-Histidine auxotroph; muta- } \\
\text { tion }\end{array}$ & YPD medium & $42^{b}$ & & $1.66^{b}$ & {$[55]$} \\
\hline Serratia marcescens SP511 & Mutation & Complete medium & $100^{b}$ & & $1.04^{\mathrm{ab}}$ & [54] \\
\hline
\end{tabular}

\footnotetext{
a Estimated from reference

b Achieved in fed-batch fermentation
} 
To construct a $C$. glutamicum strain carrying a point mutation resulting in an amino acid substitution from glycine to aspartate at position 149 of the ProB polypeptide, the $\operatorname{proB}$ gene was first deleted in the WT strain. The upstream and downstream homologous fragments of proB were amplified using PCR with primers $\mathrm{P} 1 / \mathrm{P} 2$ and P3/P4. The amplified DNA fragments were spliced using overlap extension PCR and were ligated into the suicide vector pK18mobsacB [72]. The plasmids were verified by DNA sequencing and then transformed into the WT strain through electroporation to generate the WT $\triangle$ proB strain, which was further verified by sequencing. In a second step, the mutation was introduced into the proB gene by PCR amplification with the primers $\mathrm{P} 1 / \mathrm{P} 7$ and P8/P4. The amplified DNA fragments were spliced using overlap extension PCR and ligated into the suicide vector $\mathrm{pK} 18$ mobsacB. The obtained plasmid was transformed into the WT $\triangle p r o B$ strain to generate the Pro-1 (WTproB*) strain [60]. For deletion of the putA gene, previously isolated WT genomic DNA was amplified using PCR with primers P9/P10 and P11/P12. The amplified DNA fragments were spliced using overlap extension PCR and ligated into the suicide vector pK18mobsacB. The plasmids were verified by DNA sequencing and then transformed into the Pro-1 (WTpro $B^{*}$ ) strain through electroporation to generate the Pro-2 (WTpro $B^{*} \Delta p u t A$ ) strain, which was further verified by sequencing.

To replace the native promoter of the acn gene with the promoter of $p c k$, the RBS sequence with 'AAAGGAGGA', and the start codon with ATG, three fragments corresponding to the upstream region of $a c n$, the introduced modulation element including the $p c k$ promoter and the RBS as well as the acn gene were amplified with primers P15/P16, P17/P18, and P19/P20, respectively. These fragments were spliced using overlap extension PCR and ligated into the suicide vector $\mathrm{pK} 18$ mobsacB. The plasmids were verified by DNA sequencing and then transformed into the Pro-2 (WTpro $B^{*} \Delta p u t A$ ) strain through electroporation to generate the Pro-3 (WTproB* $\mathrm{P}_{p c k}$ : $\left.: \mathrm{P}_{a c n} a c n^{*} \Delta p u t A\right)$ strain, which was further verified by sequencing. Similarly, the promotor of the acn gene was altered in Pro-2 (WTpro $B^{\mathrm{G} 446 \mathrm{~A}} \Delta p u t A$ ), which generated Pro-4 (WTproB* $\mathrm{P}_{g l y A}: \mathrm{P}_{a c n} a c n^{*} \Delta p u t A$ ) and Pro-5 (WTproB* $\mathrm{P}_{\text {eftu }}: \mathrm{P}_{a c n} a c n^{*} \Delta p u t A$ ).

The C. glutamicum and E. coli shuttle vector pXMJ19 was used for the overexpression of the $p r o B^{*}$ gene [73]. For amplification of the pro $^{*}$ gene, genomic DNA isolated from Pro-1 (WTproB* ${ }^{*}$ ) was amplified using PCR with primers P31/P32. Then, the amplified DNA fragment was ligated into the shuttle vector pXMJ19. The plasmids were verified by DNA sequencing and then transformed into the Pro- 5 (WTproB* $\mathrm{P}_{\text {eftu }}: \mathrm{P}_{a c n} a c n * \Delta p u t A$ ) strain through electroporation to generate the Pro-6 (WTproB* $\mathrm{P}_{\text {eftu }}: \mathrm{P}_{a c n} a c n * \Delta p u t A$ /
pXMJ19-pro $\left.B^{*}\right)$ strain. When needed, $0.1 \mathrm{mM}$ IPTG was added to the culture medium to induce target gene overexpression. The primers used in this study are provided in Additional file 1: Table S4.

\section{Media and cultivation}

The E. coli strains were aerobically grown at $37{ }^{\circ} \mathrm{C}$ in Luria-Bertani medium [74]. The C. glutamicum strains were cultivated in brain heart infusion (BHI) medium (37 g/L brain heart infusion with $91 \mathrm{~g} / \mathrm{L}$ sorbitol) at $30^{\circ} \mathrm{C}$ for genetic disruption and complementation [75]. As a minimal medium, CGXII medium was used with $40 \mathrm{~g} / \mathrm{L}$ glucose [76]. When necessary, antibiotics were added at the following concentrations: $50 \mu \mathrm{g} / \mathrm{mL}$ kanamycin or $20 \mu \mathrm{g} / \mathrm{mL}$ chloramphenicol for $E$. coli and $25 \mu \mathrm{g} / \mathrm{mL}$ kanamycin or $10 \mu \mathrm{g} / \mathrm{mL}$ chloramphenicol for C. glutamicum.

In the shake-flask growth experiment, C. glutamicum strains were precultured in the CGIII seed medium at $30^{\circ} \mathrm{C}$ and $200 \mathrm{rpm}$ until the $\mathrm{OD}_{600}$ reached 12. One milliliter of seed culture was inoculated in a $500-\mathrm{mL}$ baffled shake flask with $30 \mathrm{~mL}$ of CGXII minimal medium. The cells were cultured in triplicate at $30^{\circ} \mathrm{C}$ and shaken at $220 \mathrm{rpm}$. The $\mathrm{pH}$ was maintained at 7.0-7.2 via ammonia supplementation.

The fed-batch fermentation was performed in a 7.5-L bioreactor (BioFlo ${ }^{\circledR} /$ CelliGen $^{\circledR} 115$, New Brunswick, USA) with a working volume of 2 L of CGX medium containing $40 \mathrm{~g} / \mathrm{L}$ glucose. After $3 \mathrm{~h}$ of growth, $0.1 \mathrm{mmol} / \mathrm{L}$ IPTG was added for the induction of $\mathrm{P}_{t a c}$. The concentration of glucose over all of the fed-batch cultures was maintained within the range of $5 \pm 5 \mathrm{~g} / \mathrm{L}$ by supplying $800 \mathrm{~g} / \mathrm{L}$ of glucose reservoir. The glucose reservoir was fed into the fermenter at the rate of $0.2-0.5 \mathrm{~mL} / \mathrm{min}$ according to the residual glucose concentration. The temperature was maintained at $32{ }^{\circ} \mathrm{C}$ using cold water circulation. The $\mathrm{pH}$ was maintained at 6.9 via the automated addition of ammonia and $100 \mathrm{mmol} / \mathrm{L} \mathrm{H}_{3} \mathrm{PO}_{4}$. Dissolved oxygen was determined using a $\mathrm{pO}_{2}$ electrode and maintained above $30 \%$ saturation via variation of the stir speed.

\section{Analytical methods}

The glucose concentration was measured using an SBA40D biosensor analyzer (Institute of Biology of Shandong Province Academy of Sciences, Shandong, China). The cell concentration was determined by measuring the absorbance at $600 \mathrm{~nm}\left(\mathrm{OD}_{600}\right)$ using a spectrophotometer (V-1100D; Mapada Instruments, Shanghai, China). The dry cell weight (DCW) per liter was calculated using an experimentally determined formula: DCW $(\mathrm{g} / \mathrm{L})=0.27 \times \mathrm{OD}_{600}[77]$. The amino acids in the culture supernatant were determined using high-performance liquid chromatography with a Zorbax Eclipse XDB- $\mathrm{C}_{18}$ column $(4.6 \mathrm{~mm} \times 150 \mathrm{~mm}, 5 \mu \mathrm{m}$; Agilent $)$ at $40{ }^{\circ} \mathrm{C}$ and $360 \mathrm{~nm}$ after derivatization with 2,4-dinitrofluorobenzene. 
Mobile phase A was 55\% (v/v) acetonitrile, and mobile phase $\mathrm{B}$ consisted of $40 \mathrm{mmol} / \mathrm{L} \mathrm{KH}_{2} \mathrm{PO}_{4}$ at $\mathrm{pH}$ 7.0-7.2. The flow rate of the mobile phase was $1 \mathrm{~mL} / \mathrm{min}$. The organic acids in the culture supernatant were determined using high-performance liquid chromatography with a SB-Aq column $(4.6 \times 250 \mathrm{~mm} ; 5 \mu \mathrm{m}$; Agilent $)$ at $40{ }^{\circ} \mathrm{C}$ and $210 \mathrm{~nm}$. Mobile phase A was acetonitrile, and mobile phase $\mathrm{B}$ consisted of $20 \mathrm{mmol} / \mathrm{L} \mathrm{KH}_{2} \mathrm{PO}_{4}$ at $\mathrm{pH} 2.3$. The flow rate of the mobile phase was $1 \mathrm{~mL} / \mathrm{min}$.

\section{RNA preparation and quantitative RT-PCR}

Corynebacterium glutamicum strains were grown to the exponential phase in CGXII minimal medium as described above. The cells were harvested and the total RNA was isolated using an RNAprep Pure Cell/Bacteria Kit (Tiangen, China). Reverse transcription of approximately 400 ng of RNA was performed with the primers listed in Additional file 1: Table S4 using a FastQuant RT Kit (Tiangen, China). Quantitative PCR was performed using GoTaq qPCR master mix (Promega, USA) in a $20-\mu \mathrm{L}$ mixture with a LightCycler ${ }^{\circledR} 96$ Real-Time PCR System (Roche, Switzerland). The C. glutamicum rpoB gene was used as the reference gene to normalize the $g l t A$, acn, icd, $k g d, g d h$, proB, proA, and proC mRNA levels [70]. Negative controls were used in each PCR run to exclude DNA and other contaminants. The qPCR products were verified via a melting curve analysis. Data collection and analyses were conducted using LightCycler ${ }^{\circledR} 96$ software (Roche, Switzerland) with the $2^{-\Delta \Delta \mathrm{CT}}$ method [78].

\section{Additional files}

Additional file 1: Table S1. Comparison of GEM attributes among various C. glutamicum models. Table $\mathbf{S 2}$. Comparison between the in silico prediction of the genes and proteins involved in the overproduction of L-valine and L-serine with the experimental data. Table S3. Strains and plasmids used in this study. Table S4. Primers used in this study.

Additional file 2. The lumped reactions of Model $\mathrm{Cg} 2$.

Additional file 3. The reactions and metabolites of $i$ CW773.

Additional file 4: Figure S1. The central metabolic network of C. glutamicum. Fig. S2. Robustness analysis of GLU5K flux on L-proline production rate by Pro-2 and Pro-5.

Additional file 5. The flux distribution values for simulations on acetate, lactate and xylose using FBA by iCW773.

Additional file 6. Overproduction of L-lysine in WT using OptForce MUST $_{\text {I }}$ by iCW773 and Model $_{\mathrm{Cg}} 2$.

Additional file 7. Overproduction of 1,2-propanediol and isobutanol in WT using OptForce MUST $_{\text {by iCW773. }}$

Additional file 8. Overproduction of L-proline in WT using OptForce MUST $_{\text {T }}$ by iCW773 and Model $_{\mathrm{Cg}} 2$.

Additional file 9. Overproduction of L-proline in WTAputA using OptForce $_{\text {Must }}$ by iCW773 and Model $_{\mathrm{Cg}} 2$.

Additional file 10. The upper and lower bounds of the ${ }^{13} \mathrm{C}$-tracer experimental fluxes used by OptForce MUST $_{\text {T }}$ and the added reactions used in this study.

\section{Abbreviations}

$\triangle$ : deletion; ACONTa(b): aconitase; C. glutamicum: Corynebacterium glutamicum; CS: citrate synthase; DCW: dry cell weight; E. coli: Escherichia coli; FBA: flux balance analysis; FVA: flux variability analysis; GEM: genome-scale metabolic model; GLU5K: $\gamma$-glutamyl kinase; HPLC: high-performance liquid chromatography; IPTG: isopropylthio- $\beta$-D-galactopyranoside; NADPH and NADP: reduced and oxidized form of nicotinamide adenine dinucleotide phosphate, respectively; $\mathrm{OD}_{600}$ : optical density at wavelength $(\lambda) 600 \mathrm{~nm}$; ORF: open reading frame; PPP: pentose phosphate pathway; PYRC: pyruvate carboxylase; TCA: tricarboxylic acid cycle; WT: wild-type.

\section{Authors' contributions}

TW and $Y Z$ conceived the study. TW, YZ, YZ, and TT designed the study. YZ, $J C, Y Z, X S, B W, S L$, and $X C$ performed the experiments. $Y Z$, JC, and $Y Z$ performed all data analysis. YZ, YZ, and TW wrote the paper. All authors read and approved the final manuscript.

\section{Author details}

${ }^{1}$ CAS Key Laboratory of Pathogenic Microbiology and Immunology, Institute of Microbiology, Chinese Academy of Sciences, Beijing 100101, China.

${ }^{2}$ University of Chinese Academy of Sciences, Beijing 100049, China. ${ }^{3}$ Beijing University of Chemical Technology, Beijing 100029, China. ${ }^{4}$ Savaid Medical

School, University of Chinese Academy of Sciences, Beijing 100049, China.

\section{Acknowledgements}

The authors would like to thank Siu Hung Joshua Chan and Professor Pengcheng Fu for discussion with the reconstruction of iCW773.

\section{Competing interests}

The authors declare that they have no competing interests.

\section{Availability of supporting data}

Data supporting the results of the article are included within this manuscript.

\section{Consent for publication}

All authors have approved the manuscript for publication.

\section{Funding}

This work was supported by grants from National Hi-Tech Research and Development Program of China (2014AA021203), the Science and Technology Service Network Initiative of the Chinese Academy of Sciences (KFJ-STSQYZD-047 and KFJ-EW-STS-078), and National Natural Science Foundation of China $(31100074,21390202$ and 21436002).

\section{Publisher's Note}

Springer Nature remains neutral with regard to jurisdictional claims in published maps and institutional affiliations.

Received: 14 February 2017 Accepted: 22 June 2017

Published online: 30 June 2017

\section{References}

1. Becker J, Giesselmann G, Hoffmann SL, Wittmann C. Corynebacterium glutamicum for sustainable bioproduction: from metabolic physiology to systems metabolic engineering. Advances in Biochemical Engineering/ Biotechnology. Berlin: Springer;2016. P. 1-47. doi:10.1007/10_2016_21.

2. Villadangos AF, Ordonez E, Pedre B, Messens J, Gil JA, Mateos LM. Engineered coryneform bacteria as a bio-tool for arsenic remediation. Appl Microbiol Biotechnol. 2014;98:10143-52.

3. Hirasawa T, Shimizu H. Recent advances in amino acid production by microbial cells. Curr Opin Biotechnol. 2016:42:133-46.

4. Lee JY, Na YA, Kim E, Lee HS, Kim P. The actinobacterium Corynebacterium glutamicum, an industrial workhorse. J Microbiol Biotechnol. 2016;26:807-22.

5. Nguyen ADQ, Schneider J, Wendisch V. Identification of engineering targets for improving putrescine production by Corynebacterium glutamicum. New Biotechnol. 2014;31:S166-S166. 
6. Shi S, Chen T, Zhao X. Comparative transcriptome analysis for metabolic engineering. Methods Mol Biol. 2013;985:447-58.

7. Lee SH, Kang KH, Kim EY, Chae TU, Oh YH, Hong SH, Song BK, Jegals J, Park SJ, Lee SY. Metabolic engineering of Escherichia coli for enhanced biosynthesis of poly(3-hydroxybutyrate) based on proteome analysis. Biotechnol Lett. 2013;35:1631-7.

8. Becker J, Zelder O, Hafner S, Schroder H, Wittmann C. From zero to hero-design-based systems metabolic engineering of Corynebacterium glutamicum for L-lysine production. Metab Eng. 2011;13:159-68.

9. Park SH, Kim HU, Kim TY, Park JS, Kim SS, Lee SY. Metabolic engineering of Corynebacterium glutamicum for L-arginine production. Nat Commun. 2014;5:4618.

10. Lee KH, Park JH, Kim TY, Kim HU, Lee SY. Systems metabolic engineering of Escherichia coli for L-threonine production. Mol Syst Biol. 2007;3:149.

11. Kim B, Kim WJ, Kim DI, Lee SY. Applications of genome-scale metabolic network model in metabolic engineering. J Ind Microbiol Biotechnol. 2015;42:339-48.

12. Kim TY, Sohn SB, Kim YB, Kim WJ, Lee SY. Recent advances in reconstruction and applications of genome-scale metabolic models. Curr Opin Biotechnol. 2012;23:617-23.

13. Damiani AL, He QP, Jeffries TW, Wang J. Comprehensive evaluation of two genome-scale metabolic network models for Scheffersomyces stipitis. Biotechnol Bioeng. 2015;112:1250-62.

14. McCloskey D, Palsson BO, Feist AM. Basic and applied uses of genomescale metabolic network reconstructions of Escherichia coli. Mol Syst Biol. 2013:9:661.

15. Reed JL, Vo TD, Schilling CH, Palsson BO. An expanded genomescale model of Escherichia coli K-12 (iJR904 GSM/GPR). Genome Biol. 2003:4:R54.

16. Feist AM, Henry CS, Reed JL, Krummenacker M, Joyce AR, Karp PD, Broadbelt $\mathrm{L}$, Hatzimanikatis $\mathrm{V}$, Palsson BO. A genome-scale metabolic reconstruction for Escherichia coli K-12 MG1655 that accounts for 1260 ORFs and thermodynamic information. Mol Syst Biol. 2007;3:121.

17. Orth JD, Conrad TM, Na J, Lerman JA, Nam H, Feist AM, Palsson BO. A comprehensive genome-scale reconstruction of Escherichia coli metabolism—2011. Mol Syst Biol. 2011;7:535.

18. Edwards JS, Palsson BO. The Escherichia coli MG1655 in silico metabolic genotype: its definition, characteristics, and capabilities. Proc Natl Acad Sci USA. 2000;97:5528-33.

19. Mienda BS, Shamsir MS, Illias RM. Model-guided metabolic gene knockout of gnd for enhanced succinate production in Escherichia coli from glucose and glycerol substrates. Comput Biol Chem. 2016;61:130-7.

20. Liu J, Qi H, Wang C, Wen J. Model-driven intracellular redox status modulation for increasing isobutanol production in Escherichia coli. Biotechnol Biofuels. 2015;8:108.

21. Xu P, Ranganathan S, Fowler ZL, Maranas CD, Koffas MA. Genome-scale metabolic network modeling results in minimal interventions that cooperatively force carbon flux towards malonyl-CoA. Metab Eng. 2011;13:578-87.

22. Shinfuku Y, Sorpitiporn N, Sono M, Furusawa C, Hirasawa T, Shimizu H. Development and experimental verification of a genome-scale metabolic model for Corynebacterium glutamicum. Microb Cell Fact. 2009;8:43.

23. Kjeldsen KR, Nielsen J. In silico genome-scale reconstruction and validation of the Corynebacterium glutamicum metabolic network. Biotechnol Bioeng. 2009;102:583-97.

24. Chai $X$, Shang $X$, Zhang Y, Liu S, Liang Y, Zhang Y, Wen T. A novel pyruvate kinase and its application in lactic acid production under oxygen deprivation in Corynebacterium glutamicum. BMC Biotechnol. 2016;16:79.

25. Jojima T, Igari T, Moteki Y, Suda M, Yukawa H, Inui M. Promiscuous activity of $(\mathrm{S}, \mathrm{S})$-butanediol dehydrogenase is responsible for glycerol production from 1,3-dihydroxyacetone in Corynebacterium glutamicum under oxygen-deprived conditions. Appl Microbiol Biotechnol. 2015;99:1427-33.

26. Pauling J, Rottger R, Tauch A, Azevedo V, Baumbach J. CoryneRegNet 6.0-updated database content, new analysis methods and novel features focusing on community demands. Nucleic Acids Res. 2012;40:D610-4.

27. Mei J, Xu N, Ye C, Liu L, Wu J. Reconstruction and analysis of a genomescale metabolic network of Corynebacterium glutamicum S9114. Gene. 2016:575:615-22.

28. The UniProt C. UniProt: the universal protein knowledgebase. Nucleic Acids Res. 2017;45:D158-69.
29. Flamholz A, Noor E, Bar-Even A, Milo R. eQuilibrator-the biochemical thermodynamics calculator. Nucleic Acids Res. 2012;40:770-5.

30. Dominguez $\mathrm{H}$, CocaignBousquet $\mathrm{M}$, Lindley ND. Simultaneous consumption of glucose and fructose from sugar mixtures during botch growth of Corynebacterium glutamicum. Appl Microbiol Biotechnol. 1997;47:600-3.

31. Peifer S, Barduhn T, Zimmet S, Volmer DA, Heinzle E, Schneider K. Metabolic engineering of the purine biosynthetic pathway in Corynebacterium glutamicum results in increased intracellular pool sizes of IMP and hypoxanthine. Microb Cell Fact. 2012;11:138.

32. Frunzke J, Engels V, Hasenbein S, Gatgens C, Bott M. Co-ordinated regulation of gluconate catabolism and glucose uptake in Corynebacterium glutamicum by two functionally equivalent transcriptional regulators, GntR1 and GntR2. Mol Microbiol. 2008;67:305-22.

33. Dominguez H, Rollin C, Guyonvarch A, Guerquin-Kern JL, CocaignBousquet M, Lindley ND. Carbon-flux distribution in the central metabolic pathways of Corynebacterium glutamicum during growth on fructose. Eur J Biochem. 1998;254:96-102.

34. Dominguez $\mathrm{H}$, Lindley ND. Complete sucrose metabolism requires fructose phosphotransferase activity in Corynebacterium glutamicum to ensure phosphorylation of liberated fructose. Appl Environ Microbiol. 1996;62:3878-80

35. Wendisch VF, de Graaf AA, Sahm H, Eikmanns BJ. Quantitative determination of metabolic fluxes during coutilization of two carbon sources: comparative analyses with Corynebacterium glutamicum during growth on acetate and/or glucose. J Bacteriol. 2000;182:3088-96.

36. Gerstmeir R, Wendisch VF, Schnicke S, Ruan H, Farwick M, Reinscheid D, Eikmanns BJ. Acetate metabolism and its regulation in Corynebacterium glutamicum. J Biotechnol. 2003;104:99-122.

37. Baumchen C, Knoll A, Husemann B, Seletzky J, Maier B, Dietrich C, Amoabediny G, Buchs J. Effect of elevated dissolved carbon dioxide concentrations on growth of Corynebacterium glutamicum on D-glucose and L-lactate. J Biotechnol. 2007;128:868-74.

38. Litsanov B, Brocker M, Bott M. Glycerol as a substrate for aerobic succinate production in minimal medium with Corynebacterium glutamicum. Microb Biotechnol. 2013;6:189-95.

39. Rittmann D, Lindner SN, Wendisch VF. Engineering of a glycerol utilization pathway for amino acid production by Corynebacterium glutamicum. Appl Environ Microbiol. 2008;74:6216-22.

40. Kang MK, Lee J, Um Y, Lee TS, Bott M, Park SJ, Woo HM. Synthetic biology platform of CoryneBrick vectors for gene expression in Corynebacterium glutamicum and its application to xylose utilization. Appl Microbiol Biotechnol. 2014:98:5991-6002.

41. Yuan YB, Yang TH, Heinzle E. C-13 metabolic flux analysis for larger scale cultivation using gas chromatography-combustion-isotope ratio mass spectrometry. Metab Eng. 2010;12:392-400.

42. Kabus A, Georgi T, Wendisch VF, Bott M. Expression of the Escherichia colipnt $A B$ genes encoding a membrane-bound transhydrogenase in Corynebacterium glutamicum improves L-lysine formation. Appl Microbiol Biotechnol. 2007:75:47-53.

43. Bartek T, Blombach B, Lang S, Eikmanns BJ, Wiechert W, Oldiges M, Noh K, Noack S. Comparative C-13 metabolic flux analysis of pyruvate dehydrogenase complex-deficient, L-valine-producing Corynebacterium glutamicum. Appl Environ Microbiol. 2011;77:6644-52.

44. Ohnishi J, Mitsuhashi S, Hayashi M, Ando S, Yokoi H, Ochiai K, Ikeda M. A novel methodology employing Corynebacterium glutamicum genome information to generate a new L-lysine-producing mutant. Appl Microbiol Biotechnol. 2002:58:217-23.

45. Sagong HY, Kim KJ. Structural insight into dihydrodipicolinate reductase from Corybebacterium glutamicum for lysine biosynthesis. J Microbiol Biotechnol. 2016:26:226-32.

46. Xu JZ, Han M, Zhang JL, Guo YF, Zhang WG. Metabolic engineering Corynebacterium glutamicum for the L-lysine production by increasing the flux into L-lysine biosynthetic pathway. Amino Acids. 2014;46:2165-75.

47. Neuner A, Wagner I, Sieker T, Ulber R, Schneider K, Peifer S, Heinzle E. Production of L-lysine on different silage juices using genetically engineered Corynebacterium glutamicum. J Biotechnol. 2013;163:217-24.

48. Becker J, Klopprogge C, Schroder H, Wittmann C. Metabolic engineering of the tricarboxylic acid cycle for improved lysine production by Corynebacterium glutamicum. Appl Environ Microbiol. 2009;75:7866-9.

49. Becker J, Klopprogge C, Herold A, Zelder O, Bolten CJ, Wittmann C. Metabolic flux engineering of L-lysine production in Corynebacterium 
glutamicum —over expression and modification of G6P dehydrogenase. J Biotechnol. 2007:132:99-109.

50. Siebert $D$, Wendisch VF. Metabolic pathway engineering for production of 1,2-propanediol and 1-propanol by Corynebacterium glutamicum. Biotechnol Biofuels. 2015;8:91.

51. Blombach B, Riester T, Wieschalka S, Ziert C, Youn JW, Wendisch VF, Eikmanns BJ. Corynebacterium glutamicum tailored for efficient isobutanol production. Appl Environ Microbiol. 2011;77:3300-10.

52. Smith KM, Cho KM, Liao JC. Engineering Corynebacterium glutamicum for isobutanol production. Appl Microbiol Biotechnol. 2010;87:1045-55.

53. Blombach B, Schreiner ME, Holatko J, Bartek T, Oldiges M, Eikmanns BJ. (L)-Valine production with pyruvate dehydrogenase complex-deficient Corynebacterium glutamicum. Appl Environ Microbiol. 2007;73:2079-84.

54. Masuda M, Takamatu S, Nishimura N, Komatsubara S, Tosa T. Improvement of culture conditions for L-proline production by a recombinant strain of Serratia marcescens. Appl Biochem Biotechnol. 1993;43:189-97.

55. Ahn J, Ryu A, Jang H, Jung JK. Effect of growth rate on the production of L-proline in the fed-batch culture of Corynebacterium acetoacidophilum. Biotechnol Bioprocess Eng. 2004;9:326-9.

56. Jensen JVK, Wendisch VF. Ornithine cyclodeaminase-based proline production by Corynebacterium glutamicum. Microb Cell Fact. 2013;12:63.

57. Jensen JV, Eberhardt D, Wendisch VF. Modular pathway engineering of Corynebacterium glutamicum for production of the glutamate-derived compounds ornithine, proline, putrescine, citrulline, and arginine. J Biotechnol. 2015;214:85-94

58. Moxley MA, Becker DF. Rapid reaction kinetics of proline dehydrogenase in the multifunctional proline utilization a protein. Biochemistry. 2012;51:511-20.

59. Sleator RD, Gahan CGM, Hill C. Mutations in the listerial proB gene leading to proline overproduction: effects on salt tolerance and murine infection. Appl Environ Microbiol. 2001;67:4560-5.

60. Hans S, Bathe B, Thierbach G. Mutant of the proB gene from coryneform bacteria. US Patent 2009022043. 2013.

61. Theodosiou E, Frick O, Buhler B, Schmid A. Metabolic network capacity of Escherichia coli for Krebs cycle-dependent proline hydroxylation. Microb Cell Fact. 2015;14:108

62. van Ooyen J, Noack S, Bott M, Reth A, Eggeling L. Improved L-lysine production with Corynebacterium glutamicum and systemic insight into citrate synthase flux and activity. Biotechnol Bioeng. 2012;109:2070-81.

63. Baumgart M, Mustafi N, Krug A, Bott M. Deletion of the aconitase gene in Corynebacterium glutamicum causes strong selection pressure for secondary mutations inactivating citrate synthase. J Bacteriol. 2011;193:6864-73.

64. Bott M. Offering surprises: TCA cycle regulation in Corynebacterium glutamicum. Trends Microbiol. 2007;15:417-25.

65. Han SO, Inui M, Yukawa H. Effect of carbon source availability and growth phase on expression of Corynebacterium glutamicum genes involved in the tricarboxylic acid cycle and glyoxylate bypass. Microbiology. 2008;154:3073-83.

66. Thiele I, Palsson BO. A protocol for generating a high-quality genomescale metabolic reconstruction. Nat Protoc. 2010;5:93-121.

67. Schellenberger J, Que R, Fleming RMT, Thiele I, Orth JD, Feist AM, Zielinski DC, Bordbar A, Lewis NE, Rahmanian S, et al. Quantitative prediction of cellular metabolism with constraint-based models: the COBRA Toolbox v2.0. Nat Protoc. 2011;6:1290-307.

68. Becker SA, Feist AM, Mo ML, Hannum G, Palsson BO, Herrgard MJ. Quantitative prediction of cellular metabolism with constraint-based models: the COBRA Toolbox. Nat Protoc. 2007;2:727-38.

69. Ranganathan S, Suthers PF, Maranas CD. OptForce: an optimization procedure for identifying all genetic manipulations leading to targeted overproductions. PLoS Comput Biol. 2010;6:e1000744.

70. Shang X, Zhang Y, Zhang G, Chai X, Deng A, Liang Y, Wen T. Characterization and molecular mechanism of AroP as an aromatic amino acid and histidine transporter in Corynebacterium glutamicum. J Bacteriol. 2013;195:5334-42.

71. Zhang Y, Shang XL, Lai SJ, Zhang GQ, Liang Y, Wen TY. Development and application of an arabinose-inducible expression system by facilitating inducer uptake in Corynebacterium glutamicum. Appl Environ Microbiol. 2012:78:5831-8.

72. Schäfer A, Tauch A, Jäger W, Kalinowski J, Thierbach G, Pühler A. Small mobilizable multipurpose cloning vectors derived from the Escherichia coli plasmids pK18 and pK19: selection of defined deletions in the chromosome of Corynebacterium glutamicum. Gene. 1994;145:69-73.

73. Jakoby M, Ngouoto-Nkili C-E, Burkovski A. Construction and application of new Corynebacterium glutamicum vectors. Biotechnol Tech. 1999:13:437-41.

74. Sambrook J, Russell DW. Molecular cloning: a laboratory manual. 3rd ed. Cold Spring Harbor: Cold Spring Harbor Laboratory Press; 2001.

75. Kalinowski J, Bathe B, Bartels D, Bischoff N, Bott M, Burkovski A, Dusch N, Eggeling L, Eikmanns BJ, Gaigalat L, et al. The complete Corynebacterium glutamicum ATCC 13032 genome sequence and its impact on the production of L-aspartate-derived amino acids and vitamins. J Biotechnol. 2003; 104:5-25

76. Hoffmann J, Altenbuchner J. Hyaluronic acid production with Corynebacterium glutamicum: effect of media composition on yield and molecular weight. J Appl Microbiol. 2014;117:663-78.

77. Lai SJ, Zhang Y, Liu SW, Liang Y, Shang XL, Chai X, Wen TY. Metabolic engineering and flux analysis of for Corynebacterium glutamicum for L-serine production. Sci China Life Sci. 2012;55:283-90.

78. Schmittgen TD, Livak KJ. Analyzing real-time PCR data by the comparative C-T method. Nat Protoc. 2008:3:1101-8.

\section{Submit your next manuscript to BioMed Central and we will help you at every step:}

- We accept pre-submission inquiries

- Our selector tool helps you to find the most relevant journal

- We provide round the clock customer support

- Convenient online submission

- Thorough peer review

- Inclusion in PubMed and all major indexing services

- Maximum visibility for your research

Submit your manuscript at www.biomedcentral.com/submit
BioMed Central 